\title{
Surface Acoustic Bloch Oscillations, the Wannier-Stark Ladder, and Landau-Zener Tunneling in a Solid
}

\author{
M. M. de Lima, Jr., ${ }^{1 *}$ Yu. A. Kosevich, ${ }^{1,2, \dagger}$ P. V. Santos,${ }^{3}$ and A. Cantarero ${ }^{1}$ \\ ${ }^{1}$ Materials Science Institute, University of Valencia, PO Box 22085, ES46071 Valencia, Spain \\ ${ }^{2}$ Semenov Institute of Chemical Physics, Russian Academy of Sciences, ul. Kosygina 4, 119991 Moscow, Russia \\ ${ }^{3}$ Paul-Drude-Institut für Festkörperelektronik, Hausvogteiplatz, 5-7, 10117 Berlin, Germany \\ (Received 23 November 2009; revised manuscript received 11 March 2010; published 23 April 2010)
}

We present the experimental observation of Bloch oscillations, the Wannier-Stark ladder, and LandauZener tunneling of surface acoustic waves in perturbed grating structures on a solid substrate. A model providing a quantitative description of our experimental observations, including multiple Landau-Zener transitions of the anticrossed surface acoustic Wannier-Stark states, is developed. The use of a planar geometry for the realization of the Bloch oscillations and Landau-Zener tunneling allows a direct access to the elastic field distribution. The vertical surface displacement has been measured by interferometry.

DOI: 10.1103/PhysRevLett.104.165502

Bloch oscillations (BOs) of an electron in a periodic potential under a constant electric field is a paradigm quantum effect $[1,2]$. BOs of the wave-packet group velocity are the result of the interplay between the particlelike acceleration by a constant driving force and wave Bragg reflection in the periodic potential. The frequencydomain counterpart of BOs is the equidistant WannierStark ladder (WSL) of localized states in a perturbed periodic system [3]. Electron BOs and WSL have been demonstrated in a number of experiments after the advent of semiconductor superlattices (SSLs) [4]. The WSL leads to resonances of the density of electronic states which were observed for the first time in optical spectra in SSLs [5]. BOs were first observed in time-resolved optical experiments in biased SSLs as oscillations of electron wave packets [6-10], and later as a periodic motion of ensembles of ultracold atoms $[11,12]$ and Bose-Einstein condensates $[13,14]$ in optical lattices. The related high-field phenomenon, Landau-Zener tunneling (LZT) between neighboring bands across the band gap $[15,16]$, was also observed in SSLs for electrons $[17,18]$, and in optical lattices for ultracold atoms [19] and Bose-Einstein condensates [20]. Optical BOs and LZT of light waves have also been observed in time-resolved experiments [21-23].

In general, Bragg reflection can cause BOs of a wave of any nature in a lattice with a weak linear gradient of the lattice potential [24]. Considerable efforts have been made recently to observe these phenomena in the emerging field of phononics [25]. For instance, BOs and WSL states have been predicted for semiconductor multilayer solid structures based on acoustic-phonon cavities [26]. Vibration WSLs were observed in one dimensional (1D) elastic chains [27]. The existence of acoustic BOs and LZT was demonstrated in an ultrasonic superlattice made of 1D water cavities between layers of a solid polymer [28] and on macroscopic 2D phononic crystals [29]. Nevertheless, the realization of such experiments in a solid state device
PACS numbers: 63.20. $-\mathrm{e}, 43.35 .+\mathrm{d}, 63.22 .-\mathrm{m}, 68.35 .-\mathrm{p}$

fabricated with standard lithography techniques has not yet been reported for phononic structures.

In this Letter, we demonstrate that fundamental effects of quantum-wave transport in a perturbed periodic potential such as BOs, WSL, and LZT can be studied with surface acoustic waves (SAWs) in a solid. Our experimental results, including multiple LZ-like transitions between the anticrossed surface acoustic WS states, are described by a theoretical model developed by us. The used planar geometry allows the direct access to the elastic field distribution of the corresponding WS and LZ eigenstates.

In our approach, we employ SAW cavities separated by acoustic Bragg reflectors (BRs). These structures are defined by locally changing the SAW propagation properties via the deposition of thin gold stripes in-between a $\mathrm{LiNbO}_{3}$ SAW delay line (see Fig. 1). The BRs are efficient barriers, presenting high reflectivity for an incoming wave packet. This arises from the destructive interference of the properly arranged set of reflectors that creates a stop-band in the transmission spectrum for the incoming wave. Further-

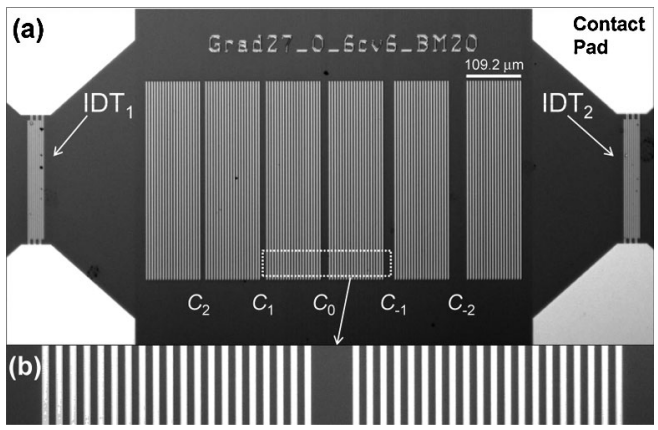

FIG. 1. (a) Photograph of a grating structure with design gradient $\delta=27 \%$. Light areas are the gold stripes and the interdigital transducers (IDTs) while dark regions correspond to the bare $\mathrm{LiNbO}_{3}$ surface. The labels $C_{n}$ indicate the graded cavities with length given by $L_{0} /(1+n \delta)$. (b) Magnified view of cavity $C_{0}$ between two Bragg reflectors. 
more, the periodic introduction of cavities inside the BR folds the frequency dispersion, producing several acoustic minibands.

The evolution of the Bloch wave vector along the axis of a perturbed periodic system is given by the Bloch "acceleration theorem" [28]:

$$
\dot{k}_{x}=-\partial \omega / \partial x, \quad v_{x}=\partial \omega / \partial k_{x},
$$

where $\omega$ is the wave angular frequency, $v_{x}$ is the group velocity of the wave packet, which is a periodic function of $k_{x} d$, and $d$ is the spatial period of the unperturbed system. In the case of weakly coupled cavities, the tight-binding approximation gives $v_{x} \propto \sin \left(k_{x} d\right)$ [28]. In this case, $\partial \omega / \partial x$ is given by the gradient of resonant frequencies $\partial \omega_{c} / \partial x$ of the coupled cavities, which plays the role of the wave driving force. The linear frequency of the wave BOs $\left(f_{B}=d\left|\partial f_{c} / \partial x\right|\right)$ in the infinite perturbed periodic system is related to the dimensionless gradient of cavities resonant frequencies $\left(\delta_{f}=d\left|\partial f_{c} / \partial x\right| / f_{C}\right)$ as $f_{B}=$ $f_{C} \delta_{f}$, where $f_{C}=\omega_{C} /(2 \pi)$ is the center frequency of the corresponding unperturbed miniband.

By using transfer-matrix (TM) calculations, we model the SAW propagation in such perturbed grating structures as the propagation of a scalar [30] acoustic plane wave through a 1D multilayer system consisting of two layers representing the metal-free and metal-coated areas in Fig. 1. The layers are assumed to have the same elastic moduli but different effective densities. The latter accounts for the reduction $\Delta V_{R}$ in SAW velocity $V_{R}$ due to massloading by the metal, which can be expressed as [31]: $\Delta V_{R} / V_{R} \sim-4 h / \lambda$ for a grating of gold stripes on a $\mathrm{LiNbO}_{3}$ substrate [32]. Here, $h$ is the thickness of the stripe and $\lambda$ is the SAW wavelength. This nonperturbative model, which is valid in the limit $h / \lambda \ll 1$ for stripes with relatively high mass density and low acoustic velocity, quantitatively accounts for the main SAW transport features using only two parameters. One is the effective thickness of the gold stripes $h$, which defines the center frequency $f_{C}$ and width of the acoustic minibands as well as the depth of the stop band produced by the BRs. The second one is the SAW velocity in the metal-free $\mathrm{LiNbO}_{3}$ layers, which is assumed to be in between the velocities of the completely metalized and the nonmetalized surfaces [33]. The latter accounts for the partial screening of the piezoelectric field induced by the metallic stripes.

The grating structures were fabricated on a $128^{\circ}$-rotated $Y$-cut $\mathrm{LiNbO}_{3}$ substrate by photolithography. The aluminum interdigital transducers (IDTs) were designed in the split-finger configuration for a center frequency of $350 \mathrm{MHz}$ (corresponding to a SAW wavelength of $11.2 \mu \mathrm{m})$ and bandwidth of $100 \mathrm{MHz}$. The gold stripes were deposited onto a $10 \mathrm{~nm} \mathrm{NiCr}$ adhesion layer. In the following, for simplicity, we will refer to such composite stripes as gold stripes and always indicate their total nominal thickness, which were 60 and $120 \mathrm{~nm}$ for the two samples investigated in this work. The gratings consist of
6 identical BRs separated by 5 metal-free regions with graded lengths defining the cavities. Each BR comprises $20 \lambda / 4$ gold stripes (corresponding to a width of $2.8 \mu \mathrm{m}$ ) separated by a $\lambda / 4$ free-surface $\mathrm{LiNbO}_{3}$ spacing. The lengths of the cavities were changed according to a constant gradient given by $L_{0} /(1+n \delta), n=-2,-1,0,1,2$. Here, $L_{0}=3 \lambda / 2=16.8 \mu \mathrm{m}$ is the length of the central cavity. This results in a set of cavities $C_{n}$ with resonant frequencies $f_{C}\left(1+n \delta_{f}\right)$, where $\delta_{f}$ is the dimensionless gradient of resonant frequencies of the coupled cavities. As will be discussed later, $\delta_{f}$ is proportional to but substantially smaller than the design gradient $\delta$. For each sample, 121 grating structures with the same $f_{C}$ were built to cover a range of $\delta$ from 0 to $30 \%$, with a grid of $0.25 \%$.

Figure 2 compares calculated [Figs. 2(a) and 2(c)] and experimental [Figs. 2(b) and 2(c)] SAW transmission amplitude for the two samples investigated. The experimental data was determined from the measurements of the $s$-parameters of the delay lines using a network analyzer. The results shown in Fig. 2(b) have been corrected for small variations in the grating properties due to nonhomogeneities over the wafer surface following the procedure described in the auxiliary material [34]. The vertical axis shows the SAW frequency while the horizontal one presents the design gradient $\delta$. The transmission in the region of the stop band defined by the BRs is associated with the miniband created by the coupled cavities. The width (in frequency) of the structures within the BR stop band reduces with Au thickness due to the reduced coupling between the cavities. There is a very good agreement between the experimental results and the TM calculations. The parameters used in the TM calculations that best fit the experimental results correspond, in the case of Fig. 2(a), to a thickness of $120 \mathrm{~nm}$ and $v_{\mathrm{SAW}}=3880 \mathrm{~m} / \mathrm{s}$ and in the case of Fig. 2(c), to a thickness of $50 \mathrm{~nm}$ and $v_{\mathrm{SAW}}=$ $3920 \mathrm{~m} / \mathrm{s}$. These values are very close to the nominal ones.

The vertical dotted lines divide Fig. 2 into three regimes of coupling between the cavities. For $\delta \lessgtr 4 \%$, the gradient is small and the transmission inside the band gap is governed by miniband wave transport. The five Fabry-Perot transmission maxima are associated with the five resonances introduced by the five coupled cavities. The WSL regime dominates for $4 \% \lesssim \delta \leqslant 8 \%$, where the resonance frequencies varies linearly with the gradient [see Fig. 2(a)]. The same feature is also observed in the measured data (more pronounced for 3 central states). Note, in addition, that there is a substantial reduction in the line widths, which arises from the reduced coupling and formation of localized ladder states in the WSL regime. For $\delta \gtrsim 8 \%$, the picture becomes more complex as states originating from higher and lower transmission bands anticross the WS states. These anticrossings induce regions of high transmission [cf. Fig. 2(a)], which are attributed to resonant LZ tunneling. The same features also appear in the experimental plots. Besides the ordinary anticrossings between two WS states [as the one indicated by $\mathrm{D}$ in 


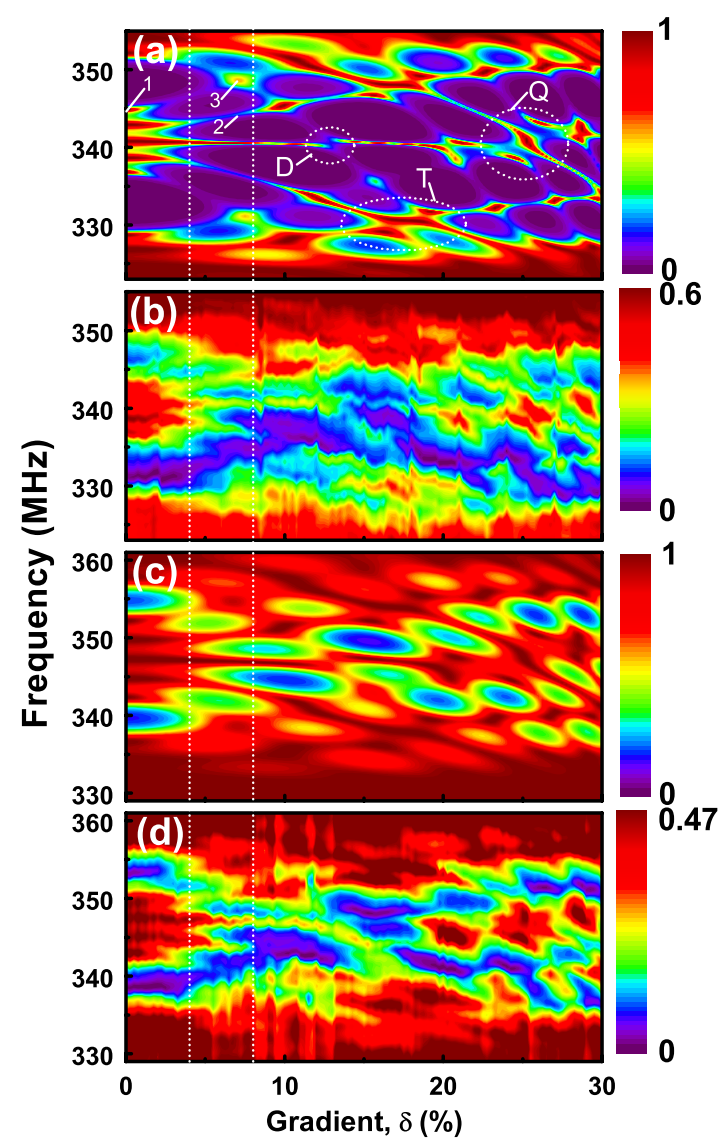

FIG. 2 (color online). Linear scale plot of SAW transmission spectra through the weakly coupled cavity structures versus the design gradient $\delta$. Plots (a) and (c) correspond to the calculations for gold stripes with effective thicknesses 120 and $50 \mathrm{~nm}$, respectively. The grating thicknesses in the experiments are nominally $120 \mathrm{~nm}$ (b) $60 \mathrm{~nm}$ (d). The vertical dotted lines indicate the region in which the ladder of surface acoustic Wannier-Stark states is seen. The letters $D, T$, and $Q$ in (a) indicate examples of ordinary, triple and quadruple anticrossings, respectively. The numbers 1, 2, and 3 in (a) point the states for which the field distributions are shown in Figs. 3(a)-3(c), respectively.

Fig. 2(a)], multiple LZ transitions can also be resolved in all these graphs: a few triple anticrossings [for example, the one indicated by $T$ ] as well as one quadruple LZT [indicated by $Q$ ].

One advantage of the planar coupled acoustic cavities relative to electronic BO systems is that the spatial distribution of the acoustic field can be directly probed. Figure 3 compares profiles of the vertical component of the surface displacement measured (left panels) by microscopic Michelson interferometry [35] with the field distribution calculated using the TM model (right panels). The overall decay in the measured profiles as one moves along the structure is a consequence of the SAW attenuation. The profiles in Fig. 3(a) for $\delta=0$ is indicated by 1 in Fig. 2(a). The corresponding elastic field is extended throughout the whole structure, having local maxima at the centers of all cavities, as expected for a state at the edge of the miniband.

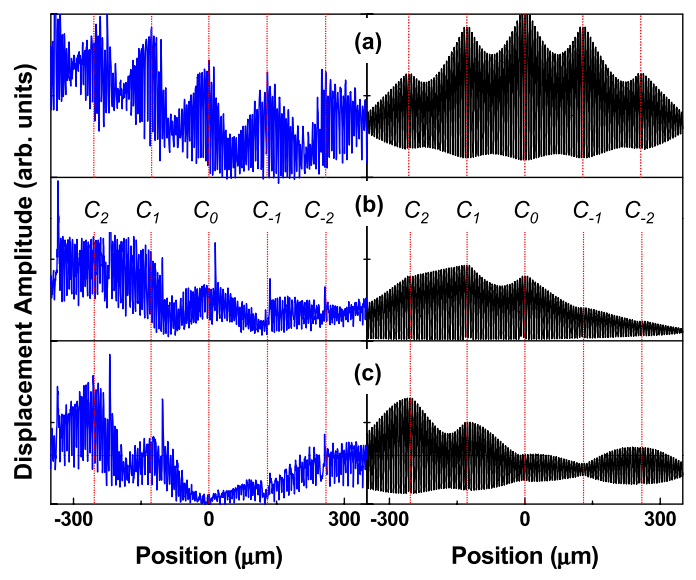

FIG. 3 (color online). Measured (left panels) and calculated (right panels) elastic field distribution along the 120-nm-thick grating structures. (a) corresponds to $\delta=0$ while (b) and (c) to $\delta=7 \%$. Their frequencies are indicated by the numbers in Fig. 2(a). The vertical lines in (a) display the position of the cavities indicated by the labels.

The results of Figs. 3(b) and 3(c) are obtained for a $\delta=7 \%$ [indicated, respectively, by numbers 2 and 3 in Fig. 2(a)]. In Fig. 3(b), the field is enhanced in the first half of the structure, demonstrating the localization associated with a WSL state. This state is related to the cavity $C_{1}$, which remains coupled to the neighbor cavities $C_{2}$ and $C_{0}$. The two lowest energetic cavities that are not coupled, therefore, present the displacement field strongly suppressed. The field distribution shown in Fig. 3(c) represents a resonant LZT, in which a state associated with the cavity $C_{2}$ anticrosses with a state associated with the cavity $C_{-2}$. The latter is originated from the upper neighbor miniband. The resulting displacement field, as compared with the one shown in Fig. 3(b), is further shifted towards the cavity $C_{2}$ while been reduced at $C_{0}$. Additionally, one can observe the effects of the anticrossing as an enhancement of the field in the region corresponding to the cavity $\mathrm{C}_{-2}$.

In order to demonstrate acoustic BOs in the WSL regime, we used the time-domain configuration of the network analyzer to measure the time-resolved transmission for different design gradients $\delta$. These results are shown in Fig. 4(a) for the sample with nominal thickness of $60 \mathrm{~nm}$. The frequency span was chosen to overlap the three strongest transmission peaks of the WSL. For each curve in Fig. 4(a), the first maximum approximately $0.3 \mu \mathrm{s}$ is associated with the SAW transit time through the $1.2 \mathrm{~mm}$ long delay line. The remaining maxima shift towards shorter times with increasing gradient $\delta$ and are associated with BOs of the SAW wave packet. Figure 4(b) compares the corresponding SAW BO frequency $f_{B}$ obtained from Fig. 4(a) with the results obtained for the sample with nominal thickness of $120 \mathrm{~nm}$. The dashed and dotted lines show the BO frequencies given by TM calculations, which approaches the asymptotic value $f_{C} \delta_{f}$ with the increase of $\delta$ cf. Eq. (1). The offset frequency coincides with the frequency separation of two neighbor Fabry-Perot reso- 


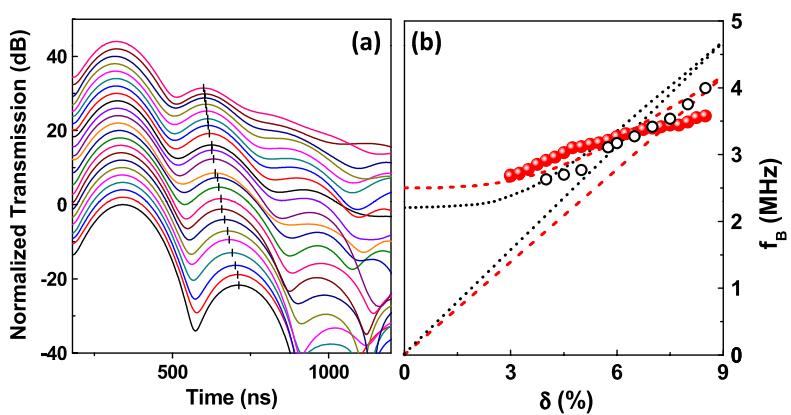

FIG. 4 (color online). (a) Time dependence of SAW transmission coefficient through the grating structures with $60 \mathrm{~nm}$ of nominal thickness for design gradients $\delta$ varying from 3.5 to $8.5 \%$. The curves are shifted upwards by $4 \mathrm{~dB}$ per $0.5 \%$ gradient. (b) Frequency of surface acoustic BOs $f_{B}$ as a function of the design gradient $\delta$ for grating structures with nominal thickness of $120 \mathrm{~nm}$ (open circles) and $60 \mathrm{~nm}$ (filled circles). Dotted and dashed lines correspond to TM calculations and their asymptotes for the infinite structure.

nances in the finite unperturbed structure and is proportional to the acoustic miniband width, which increases with the decrease of the stripe thickness [cf. Figs. 2(a), 2(c), and 4(b)]. Wider miniband width results in earlier anticrossings with the WS states originating from the neighboring acoustic minibands, which deviates the SAW BO frequency from the linear asymptotic regime, starting from smaller $\delta$ for samples with smaller stripe thickness.

Finally, a consequence of our chosen design in which the driving force is present only in the cavities, with the BRs remaining unperturbed, is the appearance of an effective acoustic field screening in our experiments. This screening substantially reduces the average driving force suffered by the SAW wave packet propagating throughout the structure. Therefore, a screening factor $s_{f}$ that relates the frequency gradient $\delta_{f}$ to the design gradient $\delta$ as $\delta_{f}=\delta / s_{f}$ can be defined, constituting an acoustic analog to the dielectric constant. This interpretation is corroborated by the fact that the TM calculations give a $s_{f}$ of approximately 7 , which is very close to the structural ratio $\left(s_{r}=7.5\right)$ between the spatial period, $d=45 \lambda / 4$, and the length of the unperturbed cavities, $L_{0}=3 \lambda / 2$.

In conclusion, we demonstrate that fundamental effects of wave transport in a perturbed periodic potential such as acoustic Bloch oscillations, Wannier-Stark ladder and Landau-Zener tunneling can be studied in solids. Furthermore, many of the exciting applications recently found for SAW technology, such as the dynamic control of the electronic and optical properties of semiconductor nanostructures for single photon sources [36], the transport of carriers for spintronic applications [37], the manipulation of coherent polariton states [38], the realization of devices for integrated photonics applications [35]; could benefit from the tunable field distribution localization investigated here. In addition, this approach is scalable and compatible with current monolithic technology.
We thank W. Seidel for the fabrication of the devices. M.M. de Lima acknowledges the Spanish Ministry of Science and Innovation for financial support through grant No. TEC2009-12075 and Yu. A. Kosevich thanks RFBR for the support through grant No. 08-02-00368.

*mmlimajr@uv.es

†ukosevich@gmail.com

[1] F. Bloch, Z. Phys. 52, 555 (1929).

[2] C. Zener, Proc. R. Soc. A 145, 523 (1934).

[3] G. H. Wannier, Rev. Mod. Phys. 34, 645 (1962).

[4] L. Esaki and R. Tsu, IBM J. Res. Dev. 14, 61 (1970).

[5] E.E. Mendez, F. Agullo-Rueda, and J. M. Hong, Phys. Rev. Lett. 60, 2426 (1988); P. Voisin et al., Phys. Rev. Lett. 61, 1639 (1988).

[6] J. Feldmann et al., Phys. Rev. B 46, 7252 (1992).

[7] K. Leo et al., Solid State Commun. 84, 943 (1992).

[8] C. Waschke et al., Phys. Rev. Lett. 70, 3319 (1993).

[9] R. Martini et al., Phys. Rev. B 54, R14 325 (1996).

[10] F. Löser, Yu. A. Kosevich, K. Köhler, and K. Leo, Phys. Rev. B 61, R13 373 (2000).

[11] M. BenDahan et al., Phys. Rev. Lett. 76, 4508 (1996).

[12] S. R. Wilkinson et al., Phys. Rev. Lett. 76, 4512 (1996).

[13] B.P. Anderson and M. A. Kasevich, Science 282, 1686 (1998).

[14] O. Morsch et al., Phys. Rev. Lett. 87, 140402 (2001).

[15] L. D. Landau, Phys. Z. Sowjetunion 2, 46 (1932).

[16] C. Zener, Proc. R. Soc. A 137, 696 (1932).

[17] H. Schneider et al., Phys. Rev. Lett. 65, 2720 (1990).

[18] B. Rosam et al., Phys. Rev. B 68, 125301 (2003).

[19] C. F. Bharucha et al., Phys. Rev. A 55, R857 (1997).

[20] C. Sias et al., Phys. Rev. Lett. 98, 120403 (2007).

[21] T. Pertsch et al., Phys. Rev. Lett. 83, 4752 (1999).

[22] H. Trompeter et al., Phys. Rev. Lett. 96, 023901 (2006).

[23] R. Sapienza et al., Phys. Rev. Lett. 91, 263902 (2003).

[24] A. Modinos et al., Physica (Amsterdam) B296, 167 (2001); Yu. A. Kosevich, Phys. Rev. B 63, 205313 (2001).

[25] M. Sigalas et al., Z. Kristallogr. 220, 765 (2005).

[26] N. D. Lanzillotti-Kimura, A. Fainstein, and B. Jusserand, Phys. Rev. B 71, 041305(R) (2005).

[27] L. Gutiérrez et al., Phys. Rev. Lett. 97, 114301 (2006).

[28] H. Sanchis-Alepuz, Yu. A. Kosevich, and J. SánchezDehesa, Phys. Rev. Lett. 98, 134301 (2007).

[29] H. Zhaojian et al., Phys. Rev. E 76, 056605 (2007).

[30] J. K. Knowles, J. Geophys. Res. 71, 5480 (1966).

[31] Yu. A. Kosevich, E. S. Syrkin, and A. M. Kossevich, Prog. Surf. Sci. 55, 59 (1997).

[32] D. H. Hurley and K. L. Telschow, Phys. Rev. B 66, 153301 (2002).

[33] G. Feuillard et al., J. Appl. Phys. 74, 6523 (1993).

[34] See supplementary material at http://link.aps.org/ supplemental/10.1103/PhysRevLett.104.165502 for the raw data as well as the details regarding the correction.

[35] M. M. de Lima, Jr. and P. V. Santos, Rep. Prog. Phys. 68, 1639 (2005)

[36] O. D. D. Couto et al., Nat. Photon. 3, 645 (2009).

[37] J. A. H. Stotz et al., Nature Mater. 4, 585 (2005).

[38] M. M. de Lima, Jr. et al., Phys. Rev. Lett. 97, 045501 (2006). 